\title{
Meeting report: The second Tianjin Forum on tumor microenvironment, Tianjin, China, June 22-24, 2012
}

\author{
Qiangzhe Zhang ${ }^{1,2}$, Lu-Yuan $\mathrm{Li}^{1,2} \bowtie$ \\ ${ }^{1}$ College of Pharmacy, Nankai University, Tianjin 300071, China \\ ${ }^{2}$ State Key Laboratory of Medicinal Chemical Biology, Tianjin 300071, China \\ $\bowtie$ Correspondence: liluyuan@nankai.edu.cn
}

The second Tianjin Forum on tumor microenvironment was held at Nankai University, Tianjin, China, from June 22 to June 24, 2012. About 300 scientists and students attended the forum, which included 28 presentations by researchers from Asia, America, and Europe on a wide variety of multidisciplinary issues regarding microenvironment impact on metastasis, tumor vasculature, inflammation and carcinogenesis, bone microenvironment, and cell-cell signaling.

Professor Jeffrey Pollard from Albert Einstein College of Medicine, New York, USA, gave the keynote speech. He discussed how monocyte and macrophage diversity promotes tumor progression and metastasis. Persuasive clinical and experimental evidence indicates that macrophages enhance malignancy at the primary site by stimulating angiogenesis, inducing tumor cell migration, invasion and intravasation and by suppressing anti-tumor immunity. At metastatic sites macrophages promote tumor cell extravasation, survival and subsequent growth. Each of these activities is stimulated by a different population of macrophages whose unique signaling pathways might represent new therapeutic targets. "Developmentally relevant" molecules such as Wnt proteins appear to play a key role. Recent studies have focused on the origin of different populations of macrophages. Lineage tracing studies indicate that the primary and metastatic tumors recruit different populations of monocytes partially explaining the macrophage diversity.

The first session of the conference focuses on microenvironmental impact on cancer development and metastasis. Professor Isaac Witz from Tel Aviv University, Israel, pointed out that tumor cells making up the primary tumor and those in metastatic lesions are genetically and phenotypically different. Using human melanoma and neuroblastoma xenograft models, he reported on molecular signa- tures of metastasis and on interactions between microenvironmental components of metastatic sites with the corresponding tumor cells. Professor Chuanyue Wu from University of Pittsburgh, Pittsburgh, USA, presented his research on integrin signaling in cancer cell survival and invasion. Cell-extracellular matrix ECM adhesion is mediated primarily by integrins, a family of $\alpha \beta$ heterodimeric transmembrane proteins. Integrins interact extracellularly with ECM proteins and intracellularly with a highly selective group of cytoplasmic proteins and thereby transmit signals across the plasma membranes. Professor Lin Zhang, also from University of Pittsburgh, talked about the role of fibulin family members as suppressors of lung cancer invasion and metastasis. His recent studies identified the fibulin family, a group of widely expressed extracellular matrix proteins that mediate cell-to-cell and cell-to-matrix communication, as suppressors of lung cancer invasion and metastasis. Two of the six fibulin family members, including fibulin-3 and fibulin-5, are frequently silenced by promoter hypermethylation in lung cancer cells and lung tumors. The inactivation of fibulin-3 and fibulin-5 is correlated with elevated MMP-7 expression and lung cancer progression, suggesting that alterations in tumor microenvironment during tumor cell invasion can be driven by epigenetic mechanisms. Professor Xi Zhan presented his work on the role of Missing in Metastasis (MIM) gene in the cell-stroma interaction and tumorigenesis. Aberrant interactions between tumor cells and a repertoire of otherwise normal cells in their local microenvironments are evident in the neoplasm derived from $B$ cells. He discussed in detail about the "missing-in-metastasis" (MIM or MTSS1) gene that encodes a protein that shapes the membrane curvature and regulates cellular polarity, endocytosis, cell motility and the actin cytoskeleton. Professor Jianfeng Chen from Shanghai 
Institute of Biochemistry and Cell Biology, Shanghai, China, spoke about regulation of integrin affinity and signaling by metal ion cluster. Integrins are a family of $\alpha / \beta$ heterodimeric adhesion metalloprotein receptors and their functions are highly dependent on and regulated by different divalent cations. Professor Zhihai Qin from Beijing Institute of Biophysics presented his research on fibroblasts, inflammation and tumor development. He analyzed multi-disciplinary interactions between tumor cells and the stromal cells, especially roles of key immune regulatory cytokines as the mediators in tumor/stroma crosstalk. Professor Gaoxiang $\mathrm{Ge}$, also from Shanghai Institute of Biochemistry and Cell Biology, discussed the role of lysyl oxidase in lung fibrosis and lung cancer progression. He pointed out that deregulation of lysyl oxidase (LOX), a copper-dependent amine oxidase responsible for the crosslinking and deposition of collagen, is closely related to a variety of human diseases, including tissue fibrosis and cancer.

The theme of session $\mathbf{2}$ is tumor vasculature. Professor Yongzhang Luo's lab, of Tsinghua University, Beijing, China, presented data on tumor stromal cell recruitment and tumor angiogenesis. They discovered that tumor released placental growth factor (PIGF) can induce myelomonocytic cell recruitment via nuclear factor of activated T cells 1 (NFAT1) and tumor necrosis factor $\alpha$ (TNF- $\alpha$ ). Professor Yulong $\mathrm{He}$ talked about regulation of lymphatic vessel growth and remodeling in development and cancer. He and colleagues employed a number of genetically modified mouse models in their studies, including a conditional VEGFR-3 knockout, a mutant model of the tyrosine kinase domain of VEGFR-3 with an inactivation point mutation (Vegfr3TKmut), and an inducible transgenic model overexpressing VEGFR-3. Professor Ruowen Ge from National University of Singapore, Singapore, discussed her work on a novel secreted angiogenesis inhibitor named isthmin. In vitro and in vivo experiments demonstrated that isthmin inhibits tumorigenesis in mice by inhibiting tumor angiogenesis. Mechanistic investigation identified a high affinity cell surface receptor and two low affinity co-receptors for isthmin on the endothelial cell surface. Professor Luyuan Li from Nankai University, Tianjin, China, presented data supporting the notion that down-modulation of TNFSF15 is a prerequisite for tumor neovascularization. He and colleagues showed that VEGF produced by cancer cells and monocyte chemotactic protein-1 (MCP-1) produced mainly by tumor-infiltrating macrophages and regulatory $T$ cells effectively inhibit TNFSF15 production by endothelial cells. Using a mouse syngeneic tumor model, removal of TNFSF15 by topical shRNA treatments prior to cancer cell inoculation could greatly facilitates angiogenesis and tumor growth. Professor Zhou Wang from University of Pittsburgh Cancer Institute, Pittsburgh, USA, showed that U19/EAF2 loss enhances pro-angiogenic effects of Von Hippel-Lindau heterozygosity on the murine liver and prostate. Professor Quansheng Zhou from Soochow University, Suzhou, China, talked about tumor cell-dominant vasculogenic mimicry. Using models of vasculogenic mimicry with human glioma stem or progenitor cells, he found that T2-tryptophanyl tRNA synthetase (T2-TrpRS) specifically inhibited tumor neovascularization through blocking the connection between two VE-cadherin molecules. Professor Lunxiu Qin from Fudan University, Shanghai, China, discussed his work on anti-inflammatory status in tumor microenvironment that facilitates the metastasis of hepatocellular carcinoma. He and colleagues constructed a refined expression signature containing 17 genes (12 Th1/Th2 cytokines, HLA-DR, HLA-DPA, ANXA1, PRG1, and CSF1) that could successfully predict both venous metastases and extrahepatic metastases by follow-up with $>92 \%$ overall accuracy. Professor Xin Zhao from Nankai University presented interesting mathematic models on pattern formation with vascular mesenchymal cells.

Session 3 of the conference focuses on bone microenvironment, hematopoiesis, and metastasis. Professor Evan Keller from University of Michigan, Michigan, USA, talked about impact of the bone microenvironment on prostate cancer progression. He pointed out that tumor cells can secrete multiple factors that promote bone resorption and osteoclastogenesis. TGF-beta directly supports tumor development and exacerbates bone resorption. Factors such as the Wnt antagonist Dickkopf-1 (DKK-1) can block Wnt-mediated maturation of marrow mesenchymal stem cells (MSC) along the osteoblast lineage. Moreover, prostate cancer cells compete with hematopoietic stem cells (HSC) for the HSC niche in the bone. Professor Xu Cao from Johns Hopkins University, Baltimore, USA, talked about coupling bone resorption with formation and marrow microenvironment. $\mathrm{He}$ and colleagues have shown that TGF $\beta 1$ recruits MSCs to the bone resorptive sites in the coupling of bone resorption with bone formation. They found that IGF-1 released from bone matrix stimulates osteoblast differentiation of MSCs for bone formation during bone remodeling. Professor Fengchun Yang from Indiana University, Indiana, USA, discussed the role of hematopoietic microenvironment in the progression of plexiform neurofibromas, focusing on a tumor suppressor gene "neurofibromatosis type 1 (NF1)". Professor Guozhi Xiao of Rush University, Chicago, USA, discussed his work on activating transcription factor 4 (ATF4), a critical transcription factor for bone remodeling. He showed that ablation of the Atf4 gene expression in mice greatly impaired skeletal vasculature and reduced microvascular density of the bone, associated with dramatically decreased expression of VEGF in osteoblasts located on trabecular and cortical bone surfaces. Professor Mingjiang $\mathrm{Xu}$, also from Indiana University, presented data to show that deletion of the Tet2 oncogene in mice leads to dysregulated hydroxylation of 5-methylcytosine and gene expression profile in hematopoietic stem cells. Professor Jian Zhang from Guangxi Medical University, Nanning, China, discussed the role of host-derived CCL2 in prostate cancer 
growth and skeletal metastasis indicating the importance of targeting both cancer cell and host cell in tumor microenvironment for cancer treatment. His lab has employed CCL2 knockouts, which were interbred to SCID mice to generate colonies of homozygous SCID/CCL2-/- mice. Professor Jianhua Wang from Jiaotong University, Shanghai, China, talked about a tumor suppressor gene named hypermethylated in cancer 1 (HIC1). His experimental results suggest that HIC1 is a new biomarker capable of preventing metastasis and providing a more effective therapeutic strategy for prostate cancer. Professor Zhinan Yin from Nankai University discussed the role of dendritic cell derived IL-27 in reshaping the tumor microenvironment. By using IL-27p28 conditional knockout mice, he and colleagues demonstrated that IL-27 is critical in protective immune response against methyl-cholanthrene induced fibrosarcoma and transplanted B16 melanoma, and that dendritic cells are the primary source of IL-27.

The last session of the conference deals with cell-cell signaling. Professor Yifan Dai presented data to support a critical role of arachidonic acid (AA)-activated mammalian target of rapamycin (mTOR) signaling in breast carcinogenesis and angiogenesis. The mTOR signaling pathway is upregulated in the pathogenesis of many cancers. His findings suggest that $A A$ is an effective intracellular stimulus of
mTOR and that AA-activated mTOR plays critical roles in angiogenesis and tumorigenesis of breast cancer. Professor Zhixiong Xiao talked about how a predominant isoform of p63, $\Delta \mathrm{Np63a}$, plays a major role in inhibiting cell migration, invasion, and cancer metastasis by controlling expression of MKP3. Professor Yu Jiang from University of Pittsburgh discussed a novel "crosstalk" between mTOR and Bcl-2. The elevated Bcl-2 expression is believed to enhance cell survival, allowing prostate cancer cells to escape apoptosis induced by androgen ablation. His recent studies showed that upregulated $\mathrm{Bcl}-2$ also stimulates the activity of mTOR, a central regulator in cell growth and proliferation. Professor Di Chen from Rush University talked about the role of cyclin D1 SUMOylation in tumorigenesis. He and colleagues identified a critical lysine residue responsible for cyclin D1 SUMOylation. Three critical SUMO Interacting motifs in the E3 ligase were also identified. His studies demonstrated that SUMOylation of cyclin D1 is critical for cyclin D1 protein stability and is related to the tumorigenesis.

\section{ACKNOWLEDGEMENTS}

This work is supported in part by a grant from The Ministry of Science and Technology of China (No. 2009CB918901 to L.Y. L.). 